\title{
COMPARAÇÃO DAS CAPACIDADES FUNCIONAIS DE MULHERES IDOSAS PRATICANTES DE EXERCÍCIOS FÍSICOS DURANTE OITO SEMANAS
}

\author{
Nelson Hilário Carneiro ${ }^{1,2}$, Carlos Onofre Vieira da Silva ${ }^{1}$ \\ Universidade do Oeste Paulista - UNOESTE. ${ }^{1}$ GEPPEFE e ${ }^{2}$ Dep. Educação Física, Presidente Prudente - SP.
}

\begin{abstract}
RESUMO
Estudos têm demonstrado que a prática regular de exercícios físicos pode auxiliar na manutenção destas capacidades. O objetivo deste estudo foi comparar as capacidades funcionais de idosas praticantes de exercícios físicos generalizados após 8 semanas de treinamento. Para tanto 14 idosas $(68,0 \pm 4,2$ anos) participaram do estudo. O grupo foi submetido ao programa de treinamento, $60 \mathrm{~min} / \mathrm{dia}, 3$ vezes/semana, durante 8 semanas. Para avaliação das capacidades funcionais foi aplicada a bateria de testes da AAHPERD, composta por testes de agilidade e equilíbrio dinâmico, flexibilidade, coordenação motora, resistência de força e resistência aeróbia geral. A significância das diferenças entre pré e pós o treinamento foi determinada por meio do Teste $t$ de Student $(p<0,05)$. Concluímos que em 8 semanas de treinamento generalizado, três vezes semanais, os resultados são mais expressáveis na coordenação, flexibilidade e na resistência aeróbia geral resultados contrários ao da agilidade e resistência de força que não obtiveram resultados significativos.
\end{abstract}

Descritores: força, treinamento, envelhecimento, exercícios físicos, capacidades funcionais

\section{COMPARISON OF THE FUNCTIONAL CAPABILITIES OF OLDER WOMEN PRACTICING PHYSICAL EXERCISES FOR EIGHT WEEKS}

\begin{abstract}
Studies have shown that regular physical exercise can help maintain these capabilities. The aim of this study was to compare the functional capabilities of older practitioners of physical exercises generalized after 8 weeks of training. 14 for both elderly $(68,0$ ? 4,2 years) participated in the study. The group underwent the training program, $60 \mathrm{~min} /$ day, 3 times / week for 8 weeks. To evaluate the functional capacity of the battery of tests AAHPERD, consisting of agility and dynamic balance, flexibility, coordination, strength endurance and overall aerobic endurance tests was administered. The significance of differences between pre and post training was determined by the Student $t$ test $(p<0.05)$. We conclude that 8 weeks of general training, three times a week, the results are expressible in coordination, flexibility and overall aerobic endurance results contrary to the agility and strength endurance who did not obtain significant results.
\end{abstract}

Keywords: strength, training, aging, exercise, functional capabilities.

\section{INTRODUÇÃO}

Com o avanço da idade, a degeneração dos sistemas biológicos provoca uma diminuição na capacidade de realização das atividades de vida diária (AVD), tais como levantar da cama, sentar, tomar banho etc ${ }^{1}$. 
O envelhecimento pode ser descrito como um conjunto de processos pelo qual todos os seres vivos vão passar, expressa a diminuição da funcionalidade dos sistemas biológicos e incapacidade de adaptação, interferindo na saúde e qualidade de vida dessa população².

Um dos sistemas que mais sofre alteração com o avanço da idade é o sistema musculoesquelético, por um processo de redução da massa magra que também é conhecido como sarcopenia. A partir dos 30 anos as pessoas começam a ter uma diminuição da massa magra e um aumento da gordura ${ }^{1}$. A perda de massa muscular principalmente as do tipo II (rápidas) resultam em uma perda considerável de força e potência, dificultando a realização das AVDs $^{1}$. Com a diminuição da força, que é de 15\% por década entre os 60 e 70 anos e a partir daí aumenta para $30 \%$ por década, contribui para o aumento do índice de quedas entre os idosos que muitas vezes pode chegar a óbito ${ }^{3}$.

Por outro lado, a idade não é um fator limitante para as pessoas realizarem atividades e exercícios físicos regulares ${ }^{1}$. Estudos têm demonstrado que a prática regular de exercícios físicos pode auxiliar na manutenção das capacidades funcionais que referem-se a agilidade e equilíbrio dinâmico, flexibilidade, equilíbrio, força, coordenação motora e resistência aeróbia para realização das AVDs, diminuindo a condição de dependência característica da senescência avançada ${ }^{4}$. Essas variáveis em particular sofrem alterações durante o processo de envelhecimento.

Vários pesquisadores têm contribuído para solucionar ou otimizar o processo de envelhecimento e a influência desse fenômeno natural sobre a qualidade de vida e a saúde dessa população ${ }^{5}$. Dessa forma o número de informações na literatura vem aumentando, fazendo com que a prática de exercícios físicos seja recomendada por organizações internacionais, como a forma mais eficaz para melhorar as condições físicas de pessoas idosas ${ }^{6}$.

A prática de exercícios físicos regulares apresenta resultados positivos, tanto no treinamento resistido como no treinamento aeróbio de pessoas idosas, sabendo que essas melhorias são menores em idosos do que em adultos jovens, entretanto qualquer melhora na condição em que se encontra uma pessoa idosa é muito importante ${ }^{7}$. As adaptações que o treinamento trás para os idosos é a mesma encontrada em adultos mais jovens. Com o treinamento regular a força também sofre adaptações significativas em relação ao desempenho nas AVDs, principalmente nos membros inferiores durante a velocidade da qual ele executa uma caminhada, com o treinamento regular a idosa ganha mais potência para realizar com mais confiança e destreza uma caminhada, o treinamento de força é importante e efetivo na diminuição da massa de gordura corporal e aumento da musculatura ativa no idoso. 
Tendo como bases essas informações, o presente estudo tem como objetivo comparar as capacidades funcionais de mulheres idosas antes e depois de serem submetidas a oito semanas de exercícios físicos generalizados.

\section{MATERIAL E MÉTODOS}

\section{Sujeitos}

Foram estudadas 14 idosas com idade de 68,0 \pm 4,2 anos, participantes do Projeto Social Athia, na cidade de Presidente Prudente. Todos os sujeitos foram inicialmente submetidos à avaliação médica que considerava características físicas e históricos de enfermidades pregressas que impedissem a participação em qualquer das atividades previstas no programa e, informados sobre a natureza, objetivos e procedimentos das avaliações e do treinamento. Foram excluídos do programa de treinamento todos aqueles indivíduos engajados em algum tipo de programa de exercício físico regular nos três meses anteriores ao estudo, intervenção cirúrgica e sintomas de demência. Todos que participaram desta pesquisa preencheram e assinaram um Termo de Consentimento Livre e Esclarecido previamente aprovado pelo Comitê de Ética em Pesquisa (CEP) da Universidade do Oeste Paulista. O numero do CEP foi 003/2008.

Para a avaliação das capacidades funcionais foi aplicada a bateria de testes da Aliança Americana para Saúde, Educação Física, Recreação e Dança (AAHPERD) ${ }^{4}$, pois se assemelha aos movimentos realizados durante as AVDs que é composta por 5 testes que avaliam as seguintes variáveis:

1) teste de agilidade e equilíbrio dinâmico (AGIL): As participantes iniciaram o teste sentadas numa cadeira com os calcanhares apoiados no solo. Ao sinal de "pronto, já" moveu-se para a direita e circundou um cone que estava posicionado a 1,50m para trás e $1,80 \mathrm{~m}$ para o lado da cadeira, retornando para a cadeira e sentando-se. Imediatamente a participante se levantou, moveu-se para a esquerda e circundou o segundo cone, retornando para a cadeira e sentando-se novamente. Isto completou um circuito. Para certificar-se de que realmente o avaliado sentou-se após retornar da volta ao redor dos cones, ele deveria fazer uma leve elevação dos pés retirandoos do solo. O avaliado deveria concluir dois circuitos completos.

2) flexibilidade (FLEX): Uma fita adesiva de $50,8 \mathrm{~cm}$ foi afixada no solo e uma fita métrica também foi afixada no solo perpendicularmente, com a marca de $63,5 \mathrm{~cm}$ diretamente colocada sobre a fita adesiva. Foram colocadas duas marcas eqüidistantes $15,2 \mathrm{~cm}$ do centro da fita métrica. A participante descalça sentou-se no solo com as pernas estendidas, os pés afastados $30,4 \mathrm{~cm}$ entre si, os artelhos apontando para cima e os calcanhares centrados nas marcas 
colocadas na fita adesiva. Com as mãos, uma sobre a outra, a participante vagarosamente deslizou as mãos sobre a fita métrica tão distante quanto pôde, permanecendo na posição final no mínimo por 2 segundos. Foram oferecidas uma tentativa de prática, seguidas de duas tentativas de teste. O resultado final foi dado pela melhor das duas tentativas anotadas.

3) coordenação motora (COO): Um pedaço de fita adesiva com $76,2 \mathrm{~cm}$ de comprimento foi fixado sobre uma mesa. Sobre a fita foram feitas seis marcas com $12,7 \mathrm{~cm}$ equidistantes entre si, com a primeira e última marca a $6,35 \mathrm{~cm}$ de distância das extremidades da fita. Sobre cada uma das seis marcas foi afixado, perpendicularmente à fita, um outro pedaço de fita adesiva com 7,6 cm de comprimento. A participante sentou-se de frente para a mesa e usou sua mão dominante para realizar o teste. Se a mão dominante fosse a direita, uma lata de refrigerante era colocada na posição 1, a lata dois na posição 3 e, a lata três na posição 5 .

4) resistência de força (RESISFOR): Foi utilizado um halter pesando $2 \mathrm{Kg}$ (peso para as mulheres). A participante sentou-se em uma cadeira sem braços, apoiando as costas no encosto da cadeira, com o tronco ereto, olhando diretamente para frente e com a planta dos pés completamente apoiadas no solo. O braço dominante deveria permanecer relaxado e estendido ao longo do corpo enquanto a mão não dominante apoiava-se sobre a coxa.

5) resistência aeróbia geral (RAG): a participante foi orientado a caminhar (sem correr) 804,67 metros numa pista de atletismo de $400 \mathrm{~m}$, o mais rápido possível. O tempo gasto para realizar tal tarefa foi anotado em minutos e segundos e transformado em segundos ${ }^{8}$.

\section{Treinamento}

O grupo (G) foi submetido ao programa de treinamento, composto por alongamento de membros superiores e inferiores, exercícios aeróbios (alguns movimentos de Ginástica Calistênica) e com pesos livres (halteres e caneleiras com pesos variados para melhor ajuste da carga), composto por meio agachamento, extensão e flexão do joelho, abdução e adução do quadril e do ombro, flexão e extensão do cotovelo, durante 8 semanas, com 3 sessões semanais, com 60 minutos, sendo 10 minutos de alongamento, 20 minutos de aquecimento com músicas (ênfase na coordenação motora e trabalho cognitivo) e 30 minutos de treinamento com pesos livres (ênfase nos membros inferiores) executando 3 séries de 12 a 15 repetições, com uma maior velocidade na fase concêntrica, com um minuto de pausa entre as séries, a carga inicial foi estipulada de forma que a voluntária conseguisse realizar 15 repetições, sendo ajustada todas as vezes que a voluntária excedesse o número de repetições por mais de duas vezes. 


\section{Análise estatística}

A significância das diferenças entre pré e pós o treinamento foi determinada por meio do Teste $t$ de Student, para amostras pareadas onde o nível de significância adotado foi de $P<0,05$.

\section{RESULTADOS}

Ao término do programa de treinamento foram coletados os dados para análise estatística, onde foi verificado um ganho significativo em três das cinco variáveis analisadas (tabela 1). As variáveis FLEX, COO e RAG obtiveram resultados significativos $(p<0,05)$, em relação a AGIL e RESFOR onde os resultados estatísticos não obtiveram significância.

Tabela 1. Valores dos testes de FLEX, COO, AGIL, RESFOR e RAG pré e pós 8 semanas de treinamento com exercícios generalizados.

\begin{tabular}{ccc}
\hline Testes & Pré & Pós \\
\hline FLEX (cm) & $57,1 \pm 9,5$ & $62,4 \pm$ \\
& & $8,5^{*}$ \\
COO (seg) & $16,7 \pm 5,9$ & $14,9 \pm$ \\
& & $4,7^{*}$ \\
AGIL (seg) & $25,5 \pm 5,2$ & $25,2 \pm 2,7$ \\
RESFOR (rep) & $30,0 \pm 5,6$ & $29,5 \pm 3,0$ \\
RAG (seg) & $565,9 \pm$ & $542,4 \pm$ \\
& 69,9 & $58,1^{*}$ \\
\hline
\end{tabular}

*p<0,05 em comparação com os resultados pré.

\section{DISCUSSÃO}

Este estudo analisou através de testes que as variáveis FLEX, COO e RAG tiveram resultados significativos ao término das oito semanas de treinamento, dessa forma o processo de envelhecimento não interferiu na adaptação aos exercícios, devido a não predominância do uso do sistema músculo esquelético como principal fator de funcionalidade e execução das tarefas exigidas. Em contrapartida as variáveis AGIL e RESFOR não obtiveram resultados significantes, pois o sistema músculo esquelético apresenta uma diminuição da sua capacidade de gerar força em velocidade, deste modo perdendo potência e a habilidade de se deslocar e desviar-se com mais facilidade. Entretanto, elas mantiveram após as oito semanas de treinamento com exercícios generalizados o mesmo nível de idoneidade para executar suas AVDs. 


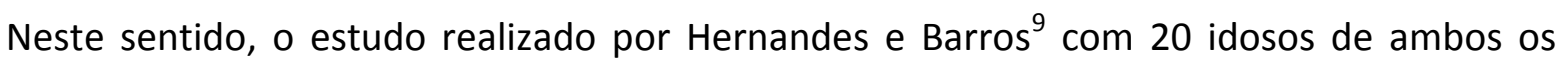
sexos na qual os submeteu a um programa de atividades físicas e educacionais durante 10 semanas, haja vista que foram avaliadas as principais capacidades físicas como a força, resistência aeróbia e flexibilidade. Os resultados obtidos foram significativos em três dos testes aplicados: caminhar/correr $800 \mathrm{~m}$, levantar-se do solo e calçar meias. Já nos testes de sentar, levantar e locomover-se, subir degraus e subir escadas, não obtiveram melhorias consideráveis. Concluindo que mesmo não tendo melhoria em todas as variáveis, ocorreu uma manutenção das capacidades meditadas, dessa forma este estudo traz considerações importantes que autenticam as informações do presente estudo.

Entretanto um estudo realizado com 60 idosas com idade média de 59,7 $\pm 5,9$ anos durante um ano, divididas em dois grupos: grupo treinado (GT) participante de um programa de atividades físicas generalizadas com três sessões semanais de uma hora de duração e o grupo controle (GC). Para avaliar a agilidade geral foi usado o teste de agilidade e equilíbrio dinâmico da bateria de testes da AAHPERD e a variável agilidade de membros superiores foi usado o teste de toque em disco do EUROFIT. O GT apresentou melhoras significativas em relação ao grupo $\mathrm{GC}^{10}$.

De acordo com Paterson as principais variáveis que se relacionam com as capacidades funcionais são a força, resistência e flexibilidade muscular, pois estas influenciam diretamente na independência, morbidade e mortalidade. A capacidade funcional pode ser descrita então como um potencial que está diretamente ligada as $\operatorname{AVDs}^{11}$, deste modo a diminuição da força pode ser considerada o principal fator limitante no processo de envelhecimento.

Para ratificar as informações acima um estudo realizado com trinta mulheres divididas em dois grupos: (G1) jovem e (G2) idoso, avaliou-se a força voluntária máxima dos músculos flexores do cotovelo (rosca unilateral) e a circunferência e dobra cutânea triciptal. Após a análise estatística, foi concluído que houve diferença significativa do G1 em relação ao G2, devido ao avanço da idade a capacidade do músculo gerar força diminui, entretanto a atividade física interfere no processo de envelhecimento ${ }^{12}$.

\section{CONCLUSÃO}

Embora os resultados não terem apresentado melhoras significativas na AGIL e RESFOR, as voluntárias mantiveram o mesmo nível de aptidão, isso devido ao pouco tempo de treinamento. Ocorreu uma melhora na FLEX, COO e RAG. Dessa forma o treinamento generalizado foi eficaz no melhoramento e manutenção das capacidades funcionais, dessa camada da população que sofre com as incapacidades e limitações características da senescência. Com esses resultados, podemos 
supor que este método de treinamento pode ser eficaz na autonomia e independência, mesmo não tendo ocorrido uma avaliação específica para analisar estas variáveis. Entretanto para obter melhores resultados sugerimos que o tempo do programa de treinamento fosse maior.

\section{REFERÊNCIAS}

1. Simão R. Fisiologia e prescrição de exercícios para grupos especiais. São Paulo: Phorte,2004.

2. Carvalho J, Mota J, Soares JM. Exercício de Força versus Exercícios Aeróbios: Tolerância Cardiovascular em Idosos. Revista Portuguesa de Cardiologia. 2003;22:1315-30.

3. Campos MA. Musculação: diabéticos, osteoporóticos, idosos, crianças, obesos. Rio de Janeiro: Sprint, 2000.

4. ZAGO, A.S.; GOBBI, S. Valores normativos da aptidão funcional de mulheres de 60 a 70 anos. Revista Brasileira de Ciência e Movimento, Brasília, v.11, n.2, p.77-86, 2003.

5. REBELATTO, Jr. et al. Influência de um programa de atividade física de longa duração sobre a força muscular manual e a flexibilidade corporal de mulheres idosas. Rev. Bras. Fisioter. V. 10, No. 1, p. 127-132, 2006.

6. Gonçalves R, Gurjão ALD, Gobbi S. Efeitos de oito semanas do treinamento de força na flexibilidade de idosos. Revista Brasileira de Cineantropometria e Desempenho Humano. 2007;9:145-53.

7. ACSM - American College of Sports Medicine. Exercise and physical activity for older adults. Med. Sci Sports Exerc 1998;30:992-1008. http://dx.doi.org/10.1097/00005768-199806000$\underline{00033}$

8. Benedetti TRB, Mazo GZ, Gobbi S, Amorim M, Gobbi LTB, Ferreira L, Hoefelmann CP. Valores normativos de aptidão funcional em mulheres de 70 a 79 anos. Revista Brasileira de Cineantropometria e Desempenho Humano 2007;9:28-36.

9. Hernandes ESC, Barros JF. Efeitos de um programa de atividades físicas e educacionais para idosos sobre o desempenho em testes de atividades da vida diária. Revista Brasileira de Ciência e Movimento. 2004;12:43-50.

10. Ferreira L, Gobbi S. Agilidade geral e agilidade de membros superiores em Mulheres de terceira idade treinadas e não treinadas. Revista Brasileira de Cineantropometria e Desempenho Humano. 2003;5:46-53.

11. Carneiro NH, Garcia Junior JR. Efeitos da prática da capoeira adaptada para terceira idade. Colloquium Vitae. 2009;1:17-24. http://dx.doi.org/10.5747/cv.2009.v01.n1.v003

12. Rogatto GP, Gobbi S. Efeitos da atividade física regular sobre parâmetros antropométricos e funcionais de mulheres jovens e idosas. Revista Brasileira de Cineantropometria e Desempenho Humano. 2001; 3:63-9. 